\title{
Analyse Des Impacts Environnementaux, Sociaux Et Economiques Des Modes De Production De Coton Conventionnel Et Biologique Au Bénin
}

\author{
Fifanou G. Vodouhe, \\ Laboratoire d'Analyses et de Recherches sur les Dynamiques Économiques \\ et Sociales Faculté d'Agronomie, Université de Parakou, Bénin
}

Gérard C. Zoundji,

Ecole de Sociologie Rurale et de Vulgarisation Agricole

Université Nationale d'Agriculture, Bénin

Hairane Yarou,

Jacob A. Yabi,

Laboratoire d'Analyses et de Recherches sur les Dynamiques Économiques et Sociales Faculté d'Agronomie, Université de Parakou, Bénin

Doi:10.19044/esj.2019.v15n36p173 URL:http://dx.doi.org/10.19044/esj.2019.v15n36p173

\section{Résumé}

Cette recherche compare les effets environnementaux, sociaux et économiques liés aux modes de production de coton conventionnel et biologique en utilisant la méthode d'analyse multicritère. Les données ayant servi à l'étude ont été collectées au sein d'un échantillon de 93 producteurs dont 34 producteurs de coton conventionnel et 59 producteurs de coton biologique. L'analyse de ces deux modes de production sur le plan environnemental, social et économique montre que la production biologique de coton reste le meilleur mode pour une agriculture durable. Les fertilisants et les produits phytosanitaires utilisés, constituent la principale différence observée au niveau des deux modes. Les producteurs de coton biologique adoptent les techniques de conservation et de restauration des sols en utilisant les fertilisants organiques et la pratique de la rotation culturale pendant que les producteurs conventionnels utilisent les pesticides et les engrais chimiques de synthèse. Ces résultats obtenus avec l'analyse multicritère peuvent être discutables et peuvent aussi varier en fonction des critères sélectionnés, de la perception des enquêtés et de la connaissance des chercheurs. Toutefois, ils peuvent servir de base dans les prises de décisions relatives à l'agriculture biologique ou conventionnelle et à la protection de l'environnement.

Mots clés : Durabilité, Pesticides, Producteurs, Coton, Bénin 


\title{
Environmental, Social and Economic Analysis of Conventional and Organic Cotton Cropping in Benin
}

\author{
Fifanou G. Vodouhe,
}

Laboratoire d'Analyses et de Recherches sur les Dynamiques Économiques et Sociales Faculté d'Agronomie, Université de Parakou, Bénin

Gérard C. Zoundji,

Ecole de Sociologie Rurale et de Vulgarisation Agricole

Université Nationale d'Agriculture, Bénin

Hairane Yarou,

Jacob A. Yabi,

Laboratoire d'Analyses et de Recherches sur les Dynamiques Économiques et Sociales Faculté d'Agronomie, Université de Parakou, Bénin

\begin{abstract}
This research compares the environmental, social and economic effects of conventional and organic cotton cropping using the multicriteria analysis method. Data were collected from a sample of 93 producers (34 conventional and 59 organic cotton producers). Results show out that organic cotton cropping is the best practice for sustainable agriculture. Fertilizers and phytosanitary products used, constitute the main difference observed in both practices. Organic cotton farmers adopt soil conservation and restoration techniques using organic fertilizers and crop rotation practices while conventional producers use synthetic chemical pesticides and fertilizers. Results show out by multicriteria analysis could be influenced by selected criteria, respondents' perception and researchers' knowledge. However, they are interesting to serve as basis for decision-making on organic or conventional agriculture and environmental protection.
\end{abstract}

Keywords: Sustainability, Pesticides, Farmers, Cotton, Benin

\section{Introduction}

Le coton joue un rôle stratégique dans l'économie des pays africains. $\mathrm{Au}$ Bénin, la filière cotonnière constitue une source de revenus pour la moitié de la population et représente $40 \%$ des recettes en devises et $13 \%$ du Produit Intérieur Brut du pays (MAEP, 2017). Elle est également la filière la mieux organisée recevant le plus d'appuis techniques et financiers de l'état compte tenu du rôle majeur qu'elle joue dans l'économie du pays (Sodjinou et al., 
2015). Cependant, cette filière est confrontée aux fortes attaques de ravageurs et dépend fortement de l'usage des pesticides chimiques de synthèses. En effet, la lutte chimique basée sur l'utilisation des pesticides chimiques demeure le principal moyen de lutte contre les ravageurs du cotonnier au Bénin. Selon New Agriculturalist (2008), $90 \%$ de l'ensemble des pesticides chimiques importés sont utilisés dans la production du coton au Bénin. La production du coton conventionnel est donc la plus grande consommatrice des pesticides et fertilisants chimiques (Traoré, 2008; Houdekon, 2010). L'utilisation excessive des intrants chimique constituait la norme et la quantité d'engrais disponible pour une campagne cotonnière au Bénin était 300 fois plus élevée que les doses recommandées par hectare (Matthess et al., 2005). Cette utilisation incontrôlée et abusive des intrants chimiques contribue certainement à l'augmentation des rendements du coton, mais constitue un danger pour la santé et pour l'environnement (Savadogo et al., 2006 ; Assogba, 2014). Ainsi, plusieurs études mettent en exergue la gravité des problèmes de santé humaine et animale, et de conservation des ressources naturelles, liés à l'utilisation des pesticides dans la production cotonnière (Westerberg, 2017 ; Glin et al., 2006).

Le changement de paradigme apparait donc comme une nécessité dans le système actuel de production cotonnière, pour faire face aux défis de santé, de préservation des écosystèmes, de la biodiversité et de l'adaptation aux changements climatiques (Pretty et al., 2011 ; Reganold et al., 2011 ; De Schutter, 2011). La promotion d'un système durable de production cotonnière s'impose. C'est dans ce contexte que le système de production du coton biologique a été introduit au Bénin vers la fin des années 1996, comme une alternative plus viable à la production du coton conventionnel (Glin et al., 2012). Ce système de production du coton gagne progressivement du terrain au Bénin. Ainsi, de 57 producteurs avec une superficie de 24 hectares en 1996 (OBEPAB, 2002), le nombre de producteurs du coton biologique était estimé à 2682 pour une superficie de 2065 hectares de terres, produisant 377 tonnes métriques de fibres biologiques en 2014 (Westerberg, 2017). Le développement progressif du système de production de coton biologique a suscité plusieurs études scientifiques. Ces études se sont surtout intéressées aux performances socio-économiques des systèmes de production biologique et conventionnelle (Berti et al., 2006 ; Zoundji, 2010 ; Westerberg, 2017 ; Dossa et al., 2018). Selon Assogba (2014), la problématique environnementale a été très peu abordée dans les études comparatives des deux systèmes de production du coton au Bénin. Il est donc urgent de combler ce gap de connaissances en vue de la durabilité de la filière cotonnière. La présente recherche vise à analyser les impacts sociaux, environnementaux et économiques des deux modes de production de coton dans la Commune de Kandi au Bénin en utilisant la méthode multicritère de prise de décision. Les 
réultats de cette étude contribueront à la prise de décisions relatives à l'agriculture biologique ou conventionnelle et à la protection de l'environnement

\section{Méthodologie Zone d'étude}

La commune de Kandi est située au Nord-Est du Bénin dans le

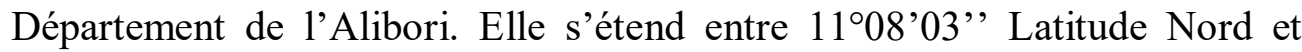
2०56'18' 'Longitude Est avec une superficie de $3421 \mathrm{~km}^{2}$ et limitée au Nord par la commune de Malanville, au Sud par la commune de Gogounou, à l'Est par la commune de Ségbana et à l'ouest par la commune de Banikoara (Dagbenonbakin et al., 2012). Cette commune est la principale zone de production du coton biologique au Bénin et la deuxième commune productrice du coton conventionnel du pays après celle de Banikoara (Dossa et al., 2018). Son climat est de type soudano sahélien avec une pluviométrie moyenne de $850 \mathrm{~mm}$ et caractérisée par une saison de pluie de Mai à Octobre et une saison sèche de Novembre à Avril. Les sols de la commune sont argileux, de type ferrugineux tropical lessivé à concrétions granito-gneissique. La végétation est composée de savane boisée, arbustive et herbacée avec des plages épineuses aux endroits soumis à une forte influence anthropique.

\section{Méthode de collecte de données}

La collecte des données a été faite en deux phases successives de Juillet à Octobre 2016. La phase exploratoire a consisté en une prise de contact, de reconnaissance et d'intégration dans le milieu d'étude. Au cours de cette phase, un guide d'entretien a été utilisé pour collecter les premières informations auprès de 12 agents du développement rural. Ces agents sont chargés de la promotion du coton conventionnel pour le compte du Secteur Communal de Développement Agricole (SCDA, Kandi) et du coton biologique pour le compte de l'Organisation Béninoise pour la Promotion de l'Agriculture Biologique (OBEPAB). En effet, l'OBEPAB est une Organisation Non Gouvernementale (ONG) installée à Kandi depuis 1996 et qui fait la promotion de la production du coton biologique. Les discussions avec ces agents qui sont des personnes ressources ont porté sur leurs interventions en faveur du développement de la filière cotonnière, les caractéristiques des deux systèmes de production du coton, et leur perception de l'influence des différentes pratiques cotonnières dans le milieu. Au cours de la seconde phase ou la phase d'enquête approfondie, la méthode d'échantillonnage raisonné a permis de sélectionner 34 producteurs de coton conventionnel dont $45 \%$ de femmes et 59 producteurs de coton biologique (45\% de femmes). La régularité dans la production cotonnière au cours des trois dernières années a été un critère de sélection des enquêtés. La collecte 
des données a été faite à l'aide d'une interview structurée portant essentiellement sur la conception de l'environnement par les producteurs, leur perception de ses fonctions et les problèmes environnementaux liés à la production cotonnière.

\section{Méthode d'analyse de données}

La méthode d'analyse multicritère (MAM) a été utilisée pour le traitement des données. En effet, la MAM est un outil décisionnel permettant d'effectuer un choix parmi plusieurs options, lorsque les bénéfices ne sont pas entièrement quantifiables en valeur financière (Faq-logistique, 2019). Elle peut donc suppléer à certaines carences de l'analyse avantages-coûts. Selon Jouffret et al. (2015), la MAM est un ensemble de méthodes visant à fournir aux décideurs des outils leur permettant de progresser dans la résolution d'un problème de décision où plusieurs points de vue, souvent contradictoires, doivent être pris en compte. Dans la pratique environnementale, beaucoup d'auteurs ont montré l'efficacité de la MAM dans la prise de décision (Mena, 2000 ; Lacasse, 2016).

Pour cette étude, les critères retenus sont une adaptation de ceux utilisés par Lacasse (2016) pour comparer les modes d'agriculture intensive, conventionnelle, raisonnée et biologique au Québec. Ces critères couvrent les trois dimensions du développement durable que sont les dimensions environnementale, sociale et économique. Pour cette étude, 18 critères ont été retenus en fonction du contexte de la production du coton dans la zone d'étude. Ces critères sont présentés dans le tableau 1. La pondération desdits critères a été faite et varie de 1 à 3 selon l'influence de chaque critère sur le choix de la pratique agricole. Les différents niveaux de la pondération faite sont les suivants :

3 : le critère est trop influent dans le choix du mode de production (biologique ou conventionnel);

2 : le critère est influent dans le choix du mode de production (biologique ou conventionnel), et

1 : le critère est peu influent dans le choix du mode de production (biologique ou conventionnel).

En s'inspirant de la boussole bernoise de la cotation des critères (OCEE, 2008), des travaux de Trepanier (2015) et de Lacasse (2016), il a été demandé aux enquêtés d'attribuer à chacun des critères, une note allant de moins deux (-2) à plus deux (+2) tel que présenté ci-dessous.

- 2 : le mode de production (biologique ou conventionnel) a un effet négatif important sur ce critère ;

- 1 : le mode de production (biologique ou conventionnel) a un faible effet négatif sur ce critère ; 
0 : le mode de production (biologique ou conventionnel) n'a pas d'effet particulier sur ce critère ;

+1 : le mode de production (biologique ou conventionnel) a un faible effet positif sur ce critère ;

+2 : le mode de production (biologique ou conventionnel) a un effet positif important sur ce critère.

Cette note attribuée par les enquêtées a été multipliée par la valeur de la pondération de chaque critère pour obtenir une cotation individuelle pour chaque critère. L'addition des notes ainsi obtenues a permis d'estimer le mode de production du coton le plus facilement applicable avec le moins d'impacts négatifs sur les trois sphères du développement durable. Au niveau de la sphère économique, seule la perception des producteurs a été prise en compte dans le travail. 
Tableau 1 : Représentation de la pondération des 18 critères servant à la réalisation de l'analyse multicritère pour la comparaison des modes de production biologique et conventionnels du coton

\begin{tabular}{|c|c|c|c|c|}
\hline Sphères & Catégories & Critères & Interprétation & Pondération \\
\hline \multirow{12}{*}{ Environnement } & \multirow{4}{*}{ Ecosystème } & $\begin{array}{l}\text { 1. Maintien de la diversité } \\
\text { biologique de l'écosystème }\end{array}$ & $\begin{array}{l}\text { Activité agricole constitue la principale préoccupation dans la vie de la } \\
\text { faune, du milieu et selon le type de système agricole appliqué }\end{array}$ & 3 \\
\hline & & 2. Couvert végétal & $\begin{array}{l}\text { Une étape importante de la pratique agricole est le défrichement qui } \\
\text { s'applique différemment selon le type de système utilisé }\end{array}$ & 3 \\
\hline & & $\begin{array}{l}\text { 3. Perte de l'habitat des } \\
\text { espèces fauniques dans le } \\
\text { milieu de culture }\end{array}$ & $\begin{array}{l}\text { Agriculture nécessite la libération d'une étendue de terre pour son } \\
\text { application en passant par le défrichement et le labour }\end{array}$ & 2 \\
\hline & & $\begin{array}{l}\text { 4. Dynamique / } \\
\text { conservation de } \\
\text { l'écosystème }\end{array}$ & $\begin{array}{l}\text { Pratique agricole change le milieu de culture et l'aire protégée autour de } \\
\text { laquelle elle est appliquée }\end{array}$ & 2 \\
\hline & \multirow{3}{*}{ Atmosphère } & 5. Emission d'azote & $\begin{array}{l}\text { Activité agricole est la principale source d'ammoniac atmosphérique qui } \\
\text { est en partie produite par l'application d'engrais et l'utilisation des } \\
\text { pesticides. Ainsi, le mode de culture utilisé a une influence. }\end{array}$ & 2 \\
\hline & & $\begin{array}{l}\text { 6. Emission de gaz à effet } \\
\text { de serre }\end{array}$ & L’émission des GES est actuellement au cœur des débats politiques & 3 \\
\hline & & 7. Emission de pesticides & $\begin{array}{l}\text { Utilisation de pesticides constitue une préoccupation majeure de la } \\
\text { société actuelle et les effets qui y sont reliés sont bien démontrés. }\end{array}$ & 3 \\
\hline & \multirow[t]{3}{*}{ Sol } & 8. Erosion des sols & $\begin{array}{l}\text { Erosion des sols agricoles survient naturellement, et ceci, } \\
\text { indépendamment du mode de culture agricole pratiqué. Cependant, } \\
\text { certaines pratiques agricoles érodent plus le sol. }\end{array}$ & 3 \\
\hline & & $\begin{array}{l}\text { 9. Gestion de la fertilité des } \\
\text { sols }\end{array}$ & Mode de culture influence la durabilité et la qualité du sol & 3 \\
\hline & & $\begin{array}{l}\text { 10. Travail / pression sur } \\
\text { les terre de culture }\end{array}$ & $\begin{array}{l}\text { Certains modes de cultures maximisent ou limitent le travail du sol, ce } \\
\text { qui peut influencer la qualité du sol. }\end{array}$ & 3 \\
\hline & Eau & 11. Epandage de pesticides & $\begin{array}{l}\text { Epandage de pesticides constitue une préoccupation majeure de la } \\
\text { société actuelle. Bien souvent, une partie des pesticides appliqués dans } \\
\text { les champs se retrouvent dans les plans d'eau et leurs effets sont bien } \\
\text { réels. }\end{array}$ & 2 \\
\hline & Social & $\begin{array}{l}\text { 12. Gestion l'espace } \\
\text { cultivable }\end{array}$ & $\begin{array}{l}\text { Choix de l'espace sur lequel établir un mode de culture donné dépend } \\
\text { largement des types de cultures pratiquées par les autres producteurs du } \\
\text { milieu. }\end{array}$ & 3 \\
\hline
\end{tabular}




\begin{tabular}{|c|c|c|c|c|}
\hline \multirow[t]{2}{*}{ Social } & & $\begin{array}{l}\text { 13. Temps d'application de } \\
\text { la technologie }\end{array}$ & $\begin{array}{l}\text { Métier de producteur agricole exige de nombreuses heures de travail qui } \\
\text { varient en fonction des pratiques agricoles utilisées. }\end{array}$ & 2 \\
\hline & Agriculteur & 14. Santé & $\begin{array}{l}\text { Certaines pratiques agricoles nécessitent une manipulation plus } \\
\text { importante de produits chimiques et amènent ainsi, un risque plus élevé } \\
\text { d'intoxication pour le producteur ou l'ouvrier agricole. }\end{array}$ & 3 \\
\hline \multirow{4}{*}{ Economie } & Consommateur & 15. Création d'emplois & $\begin{array}{l}\text { Création et le maintien des emplois sont constamment à préconiser, } \\
\text { puisque les usines d'égrenage constituent en grande partie, la base de } \\
\text { notre société }\end{array}$ & 3 \\
\hline & \multirow{3}{*}{$\begin{array}{l}\text { Perception de } \\
\text { l'agriculteur }\end{array}$} & $\begin{array}{l}\text { 16. Perception des coûts } \\
\text { liés à la vente des récoltes } \\
\text { (FCFA/tonne) }\end{array}$ & $\begin{array}{l}\text { Plus les agriculteurs obtiennent un prix de vente élevé pour leurs } \\
\text { récoltes, plus les rendements économiques de l'exploitation agricole } \\
\text { vont augmenter }\end{array}$ & 2 \\
\hline & & $\begin{array}{l}\text { 17. Perception des coûts } \\
\text { liés à l'achat des semences }\end{array}$ & $\begin{array}{l}\text { Agriculteurs doivent se procurer annuellement d'intrants agricoles. Plus } \\
\text { le coût des intrants est élevé, plus cela entraine des dépenses } \\
\text { supplémentaires et risque de diminuer la rentabilité financière de } \\
\text { l'entreprise agricole. }\end{array}$ & 2 \\
\hline & & $\begin{array}{l}\text { 18. Perception de } \\
\text { l'agriculteur sur le } \\
\text { rendement agricole }\end{array}$ & $\begin{array}{l}\text { Rendement des cultures est proportionnel aux potentiels de rentabilité. } \\
\text { Une culture est plus productive, plus la rentabilité de l'entreprise a un } \\
\text { potentiel élevé. }\end{array}$ & 3 \\
\hline
\end{tabular}

Source: Adaptation de Lacasse, 2016 


\section{Résultats et discussion}

\section{Caractéristiques démographiques et socio-économiques des producteurs}

L'échantillon de recherche est composé de $55 \%$ de producteurs de sexe masculin $(35 \%$ de producteurs de coton biologique et $20 \%$ de producteurs de coton conventionnel) et $45 \%$ de sexe féminin ( $29 \%$ de producteurs de coton biologique et $16 \%$ de producteurs de coton conventionnel).

L'âge moyen des producteurs de coton biologique dans la zone d'étude est 32 ans $( \pm 09$ ans) et de 38 ans $( \pm 07$ ans) pour les producteurs conventionnels. L'âge moyen des enquêtés montre que les jeunes sont plus engagés dans la production cotonnière dans la Commune de Kandi et les superficies moyennes qu'ils emblavent sont de 0,50 hectare pour la production biologique et de 5 hectares $( \pm 02$ ha) pour la production conventionnelle. Les producteurs justifient cette différence de superficie observée par le fait que le mode de production biologique est plus contraignant que celui conventionnel. Ainsi, les producteurs du coton biologique préfèrent se concentrer sur une superficie réduite afin de bien l'entretenir et obtenir de meilleurs rendements.

\section{Impact environnemental des modes de production de coton sur l'écosystème et l'atmosphère}

Il ressort de la Figure 1 et du Tableau 2 que selon les producteurs, l'agriculture biologique contribue plus au maintien de la biodiversité que l'agriculture conventionnelle qui favorise, plus la destruction du couvert végétal à travers la coupe des espèces végétales. Cette perception des acteurs s'explique par la conquête de nouvelles terres pour l'expansion de la production cotonnière dans la zone. Les producteurs ont également souligné que la conservation des espèces végétales en occurrence les ligneux, est une pratique fondamentale de l'agriculture biologique qui permet de fournir un habitat aux oiseaux et autres ennemis naturels des insectes ravageurs. Au niveau de la destruction de l'habitat de la faune, les producteurs des deux modes de production soutiennent que la production conventionnelle détruit plus l'habitat de la faune que celle biologique. En effet, pour ces derniers, le succès de la production conventionnelle amène les producteurs chaque année à défricher de nouvelles terres alors que dans le mode biologique, l'agriculteur reste plus longtemps sur la même terre. Compte tenu des exigences de l'agriculture biologique, les producteurs préfèrent souvent ne pas emblaver une grande superficie de terre. Ainsi, ce mode de production limite le défrichement de nouvelles terres qui entraîne la disparition de l'habitat des espèces fauniques.

L'analyse $\mathrm{du}$ critère lié à la dynamique de conservation de l'écosystème ressort que la production biologique assure mieux la conservation de l'écosystème. Selon les producteurs, l'écosystème est assez dégradé après quelques années de production conventionnelle. En se référant 
au Tableau 2 on observe que l'écosystème a obtenu la plus faible cotation (17) pour le coton conventionnel et la plus forte cotation $(+15)$ pour celui biologique. Ainsi, la production biologique assure mieux la conservation de l'écosystème que celle conventionnelle.

Les producteurs ont également observé que le mode conventionnel de production de coton émet plus d'azote que celui biologique. En effet, la production de coton conventionnel nécessite l'apport des intrants chimiques. L'utilisation des engrais chimiques de synthèse induit la chaleur $(\mathrm{CO} 2)$ à travers la destruction de la fertilité de l'espace de culture et affaiblit à petit cout l'état du sol mise en culture. Par contre, le mode biologique de production de coton est un système plus naturel mais consiste à travailler le sol pour lui permettre de s'enrichir par lui-même. Pour ce qui est de l'émission de particules dans l'atmosphère, les producteurs de coton de la commune de Kandi estiment que le mode conventionnel de production de coton est plus à la base de ce phénomène. En effet, ces derniers se sont référés à l'odeur envaillissante des insecticides dans l'atmosphère lors des traitements des champs de coton conventionnel. Ces derniers mesurent en réalité le poids des dangers que découle l'utilisation des insecticides. Concernant l'émission de Gaz à Effet de Serre, les producteurs soutiennent que le mode conventionnel de production du coton émet plus de gaz que celui biologique. Ce résultat est en lien avec la destruction du couvert végétal, imputable à l'agriculture conventionnelle selon les enquêtés. En effet, le défrichement et les pratiques de brulis dans la production conventionnelle de coton, conduisent à l'émission de gaz. Mais les exigences du mode biologique interdisent de brûler les débris végétaux lors de la préparation du sol. Les producteurs sont obligés d'enfouir les débris végétaux dans le sol afin de permettre leur dégradation et leur incorporation comme matière organique.

$\mathrm{Au}$ regard de tout ce qui précède, le mode de production biologique maintient plus la biodiversité, participe moins à la destruction du couvert végétal, protège mieux l'habitat des espèces animales et assure une dynamique positive pour l'écosystème du milieu de production, comparativement au mode conventionnel qui a obtenu une faible cotation (-16). Il est prouvé que l'agriculture biologique est l'un des modes de production durable ayant pour objectif la conservation de l'écosystème (Mader et al., 2002 ; Lampkin et Measures, 2001 ; Reganold et al., 1990). 


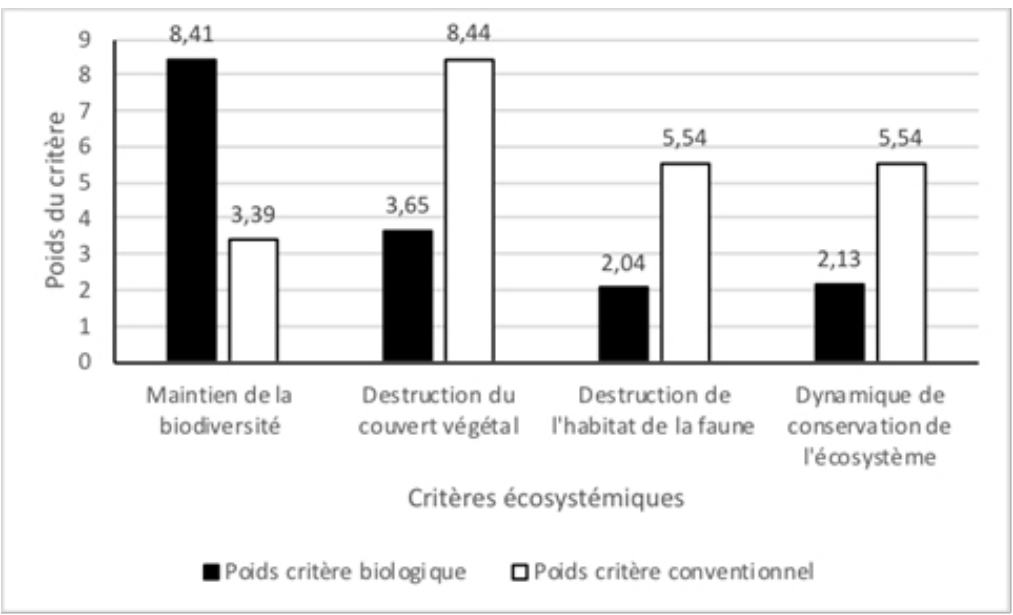

Figure 1 : Analyse comparée des effets sur l'écosystème des modes de production biologique et conventionnel du coton

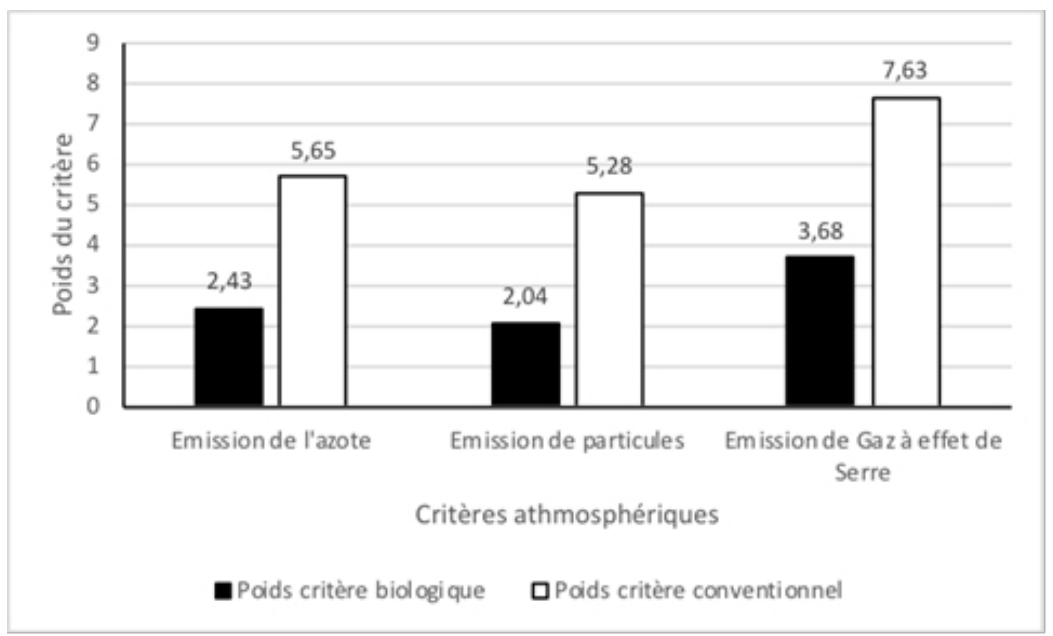

Figure 2 : Analyse comparée des effets sur l'atmosphère des modes de production biologique et conventionnel du coton 
Tableau 2 : Evaluation des deux modes de production selon la cotation faite

\begin{tabular}{|c|c|c|c|c|c|}
\hline Sphères & Catégories & $\begin{array}{ll}\text { Critères } \\
\end{array}$ & Pondération & Conventionnelle & Biologique \\
\hline \multirow{15}{*}{ Environnement } & \multirow{4}{*}{ Ecosystème } & 1. Maintien de la diversité biologique de l'écosystème & 3 & -2 & +2 \\
\hline & & 2. Couvert végétal & 3 & -1 & +1 \\
\hline & & 3. Perte de l'habitat des espèces fauniques dans le milieu de culture & 2 & -2 & +1 \\
\hline & & 4. Dynamique / conservation de l'écosystème & 2 & -2 & +2 \\
\hline & \multirow{4}{*}{ Atmosphère } & Total & & -17 & +15 \\
\hline & & 5. Emission d'azote & 2 & -2 & +1 \\
\hline & & 6. Emission de gaz à effet de serre & 3 & -2 & +1 \\
\hline & & 7. Emission de pesticides & 3 & -2 & +2 \\
\hline & \multirow{4}{*}{ Sol } & Total & & -16 & +11 \\
\hline & & 8. Erosion des sols & 3 & -1 & +1 \\
\hline & & 9. Gestion de la fertilité des sols & 3 & -2 & +2 \\
\hline & & 10. Travail / pression sur les terre de culture & 3 & -2 & -1 \\
\hline & & Total & & -15 & +6 \\
\hline & Eau & 11. Epandage de pesticides & 2 & -2 & +2 \\
\hline & Total & & & -4 & +4 \\
\hline \multicolumn{3}{|c|}{ Total de la sphère environnement } & & -52 & +36 \\
\hline \multirow{5}{*}{ Social } & Social & 12. Gestion l'espace cultivable & 3 & -1 & +2 \\
\hline & \multirow{4}{*}{ Agriculteur } & Total & & -3 & +6 \\
\hline & & 13. Temps d'application de la technologie & 2 & +1 & -2 \\
\hline & & 14. Santé & 3 & -2 & +2 \\
\hline & & Total & & -4 & +2 \\
\hline \multicolumn{2}{|c|}{ Total de la sphère sociale } & & & -7 & +8 \\
\hline \multirow{8}{*}{ Economie } & Consommateur & 15. Création d'emplois & 3 & +1 & -1 \\
\hline & \multirow{4}{*}{$\begin{array}{l}\text { Perception de } \\
\text { l'agriculteur }\end{array}$} & Total & & +3 & -3 \\
\hline & & 16. Perception des coûts liés à la vente des récoltes (FCFA/tonne) & 2 & -2 & +2 \\
\hline & & 17. Perception des coûts liés à l'achat des semences & 2 & -1 & -1 \\
\hline & & 18. Perception de l'agriculteur sur le rendement agricole & 3 & +1 & -2 \\
\hline & \multirow{2}{*}{\multicolumn{2}{|c|}{ Total de la sphère économie }} & & -3 & -4 \\
\hline & & & & 0 & -7 \\
\hline & & TOTAL GENERAL & & -59 & +37 \\
\hline
\end{tabular}




\section{Impact environnemental des modes de production de coton sur le sol et l'eau}

Le sol est le principal facteur de production en agriculture. Il représente de ce fait l'un des facteurs importants à considérer lorsqu'il s'agit d'analyser les effets environnementaux de l'agriculture. La Figure 3 présente les effets sur le sol de trois paramètres identifiés (érosion du sol ; durée de fertilisation $\mathrm{du}$ sol et pression physique due aux pratiques agricoles) pour apprécier l'impact des pratiques agricoles biologique et conventionnelle de production cotonnière.

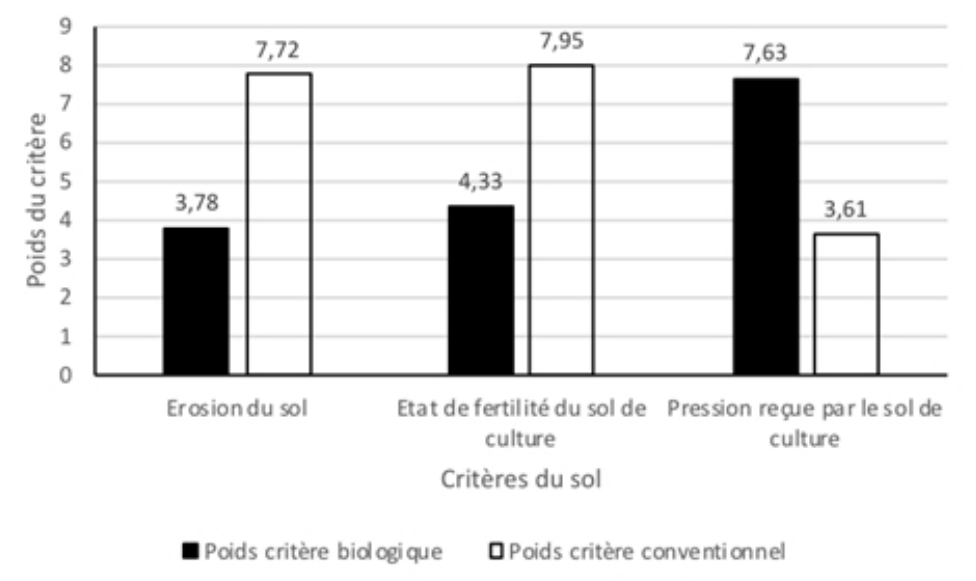

Figure 3 : Analyse comparée des effets sur le sol des modes de production biologique et conventionnel du coton

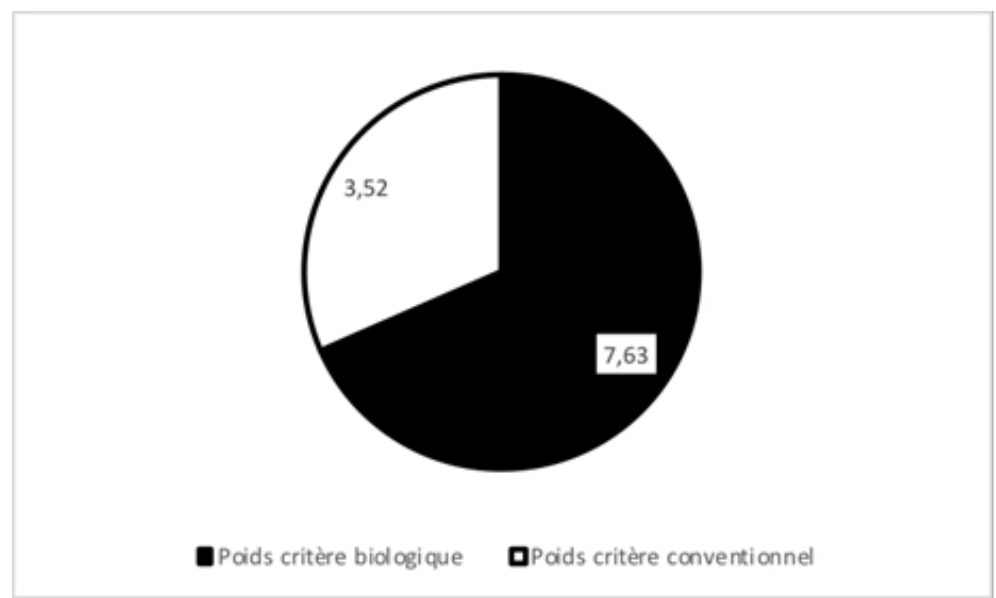

Figure 4 : Analyse comparée des effets sur l'eau des modes de production biologique et conventionnel du coton

Il ressort de la Figure 3 et du Tableau 2 que la production biologique détruit moins le sol que la production conventionnelle à travers l'utilisation excessive des produits chimiques de synthèse. En effet, la production 
cotonnière généralement nécessite une abondante fertilisation du sol. Or le mode de production biologique est un mode de production qui consiste à travailler physiquement le sol sans apport d'intrant chimique comme fertilisant. Toutefois, pour une bonne croissance du végétal, un apport d'engrais organique est fait, afin de produire sans épuiser l'espace de culture. En ce qui concerne le maintien de la fertilité du sol, il est remarqué une réduction de la vitesse d'épuisement de la fertilité des sols sous production biologique. Ce constat fait par les producteurs pourrait s'expliquer par les principes biologiques de production de coton. Ce résultat vient confirmer ceux de Oehl et al. (2004) et Pimentel et al. (2005) qui ont prouvé l'impact positif des pratiques biologiques sur le maintien du sol en production biologique. En effet, le maintien sur une période plus ou moins longue du niveau de fertilité des sols en production biologique pourrait s'expliquer par l'enfouissement de la matière organique ; un principe cher au mode de production biologique. De plus, la non utilisation d'engrais chimique permet de rehausser la présence de micro-organisme dans le sol ; ce qui favorise une bonne décomposition des matières organiques. Cette activité des micro-organismes est renforcée par la non utilisation du feu pour brûler la matière organique. Ce résultat corrobore ceux de Oehl et al. (2004) et Crowder et al. (2012) qui ont ressorti les effets du feu de végétation comme l'un des facteurs limitant dans la durabilité de conservation de l'espace de culture. Ainsi, la production biologique maintient mieux le niveau de fertilité du sol comparativement à la production conventionnelle.

Cependant, au niveau du critère concernant la pression dans les espaces de culture, il ressort qu'il y a une diminution apaisée de dégât dans la production conventionnelle. Les résultats de cette étude montrent que le mode de production biologique impacte plus physiquement le sol comparativement au mode de production conventionnelle. On en déduit donc que la production biologique assure peu la conservation de la fertilisation $\mathrm{du}$ sol comparativement à la production conventionnelle. Dans l'ensemble des critères de sol, on retient des producteurs des deux modes de production que la production conventionnelle est responsable des menaces au niveau du sol à savoir l'érosion du sol ; la durabilité de la fertilité du sol mais réduit aussi la pression que reçoivent les espaces mis en culture.

Pour analyser l'impact des modes de production sur l'eau, c'est le critère épandage de pesticide qui a été considéré. La Figure 4 présente l'impact perçu sur l'eau de l'épandage de pesticides en agriculture par les producteurs de coton. On observe que le mode d'épandage de pesticide dans le système conventionnel de production cotonnière est à la base de la contamination des plans d'eau. En effet, dans les zones de production, il est courant d'apprendre que suite au traitement du coton par les pesticides chimiques de synthèse, les animaux ou encore les populations humaines se sont intoxiquées en buvant 
l'eau des cours d'eau. Cette situation s'explique par le lessivage des feuilles par l'eau de pluie, suite à la pulvérisation et leur ruissellement vers les cours d'eau.

On retient que la production biologique de coton assure la conservation de l'environnement au regard des paramètres considérés. Les mêmes producteurs ont estimé que la production biologique de coton reste sans doute un système de production d'intérêt environnemental, vu que son application crée moins de problèmes environnementaux comparativement à la production conventionnelle de coton. Ces résultats confirment ceux de Vognan et al. (2017) qui montrent que le mode de production du coton conventionnel dépend essentiellement des produits chimiques de synthèse.

\section{Impact social des modes de production de coton}

La Figure 5 présente les contraintes que les producteurs perçoivent de la gestion de l'espace de culture dans les deux modes de production. La pratique de l'agriculture biologique a été identifiée comme trop contraignante, notamment le choix de l'espace de culture. En effet, avant de retenir un champ pour la culture du coton biologique, il faudrait faire valider le choix de la parcelle par les responsables en charge de la production biologique. Le champ doit respecter certaines conditions telles que l'isolement avec les champs voisins, l'accessibilité etc. qui ne sont pas toujours faciles à obtenir surtout que certaines d'entre elles dépendent du voisinage. Or si le champ qui reçoit la culture ne respecte pas les dispositions requises, le coton qui en sortira ne sera pas homologué et donc ne pourra pas être vendu comme biologique.

La Figure 6 présente la perception des agriculteurs du temps à consacrer à l'application de chaque mode de culture ainsi que l'effet de chaque mode sur la santé des acteurs. La production biologique du coton se révèle être celle qui nécessite plus de temps de travail selon les producteurs. Les travaux réalisés par Dossa et al. (2018) au Bénin et Vognan et al. (2017) au BurkinaFaso, confirment que le système biologique nécessite plus de temps de travail que le système conventionnel. En effet, l'apport organique et le traitement phytosanitaire en production biologique nécessite de la part du producteur beaucoup de temps de préparation. Contrairement à la culture conventionnelle pour laquelle les producteurs reçoivent des engrais et pesticides prêts à être utilisés, il revient aux producteurs de coton biologique de préparer eux-même son bio-pesticide et d'apporter de la fumure organique dans son champ. Ces différentes opérations demandent plus de temps de travail et limitent la pratique de l'agriculture biologique. Cependant, dans le mode conventionnel de production, la manipulation des pesticides chimiques de synthèse cause des problèmes de santé aux producteurs (cas d'intoxication dus aux mauvaises utilisations). Ainsi, la production biologique de coton, quoique pénible en 
termes de charges de travail, reste socialement intéressante avec moins d'impact négatif sur la santé des agriculteurs.

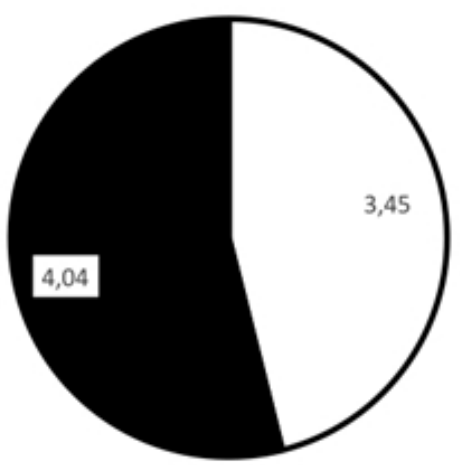

DPoids critère biol ogi que

Poids critère conventionnel

Figure 5 : Analyse comparée des effets sur le social des modes de production biologique et conventionnel du coton

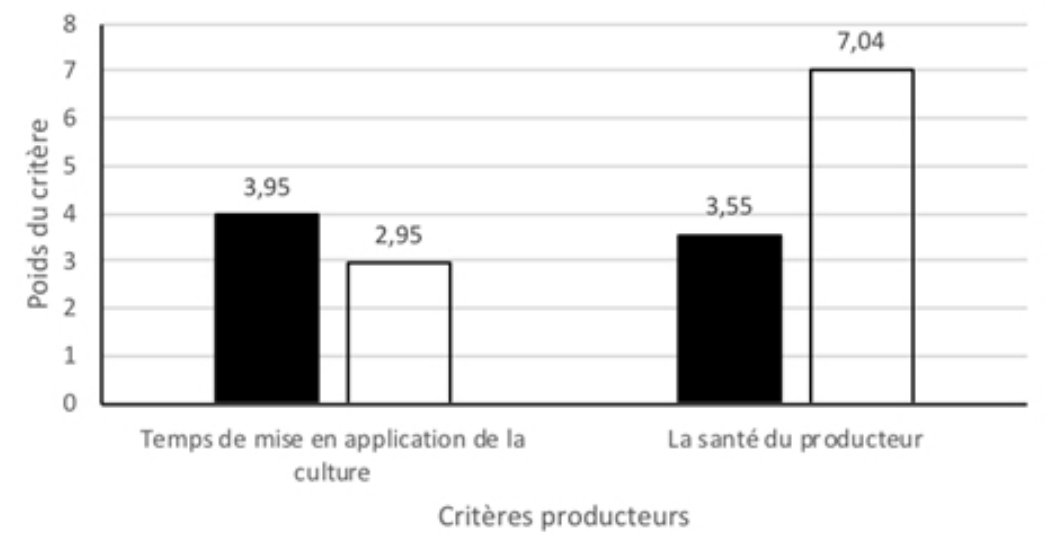

- Poids critère biologi que

口Poids critère conventionnel

Figure 6 : Analyse comparée des effets sur la santé des modes de production biologique et conventionnel du coton

\section{Impact économique des modes de production de coton}

La Figure 7 montre que la production biologique crée plus d'emploi que la production conventionnelle. Dans le même temps, les coûts de production en agriculture conventionnel sont plus élevés qu'en agriculture biologique (Figure 8). En effet, il existe une grande différence au niveau des intrants utilisés dans les deux modes de production. Selon les agriculteurs, les intrants utilisés en production conventionnelle sont plus coûteux que ceux utilisés en agriculture biologique. On en déduit que le système de production 
biologique ne nécessite pas trop de moyen financier pour son application, comparativement au mode de production conventionnelle où le moyen financier tient une part non négligeable. A cet effet, la production biologique pourrait donc être considérée comme une option politique pertinente pour soutenir les petits producteurs agricoles.

Concernant le revenu tiré des deux modes de production, la Figure 9 permet de ressortir que le mode de production biologique présente un avantage économique comparativement au mode conventionnel de production. Par ailleurs, les producteurs des deux systèmes expliquent cela par le fait que la tonne de coton biologique est plus chère que celle du coton conventionnel (312 $000 \mathrm{~F}$ la tonne de coton biologique contre $260000 \mathrm{~F}$ pour la tonne de coton conventionnel (OBEPAB, 2013). Ce résultat est contraire à celui de Pimentel et al. (2005) et pourrait s'expliquer par le fait que les producteurs ne tiennent pas compte de certains coûts liés à la production biologique du coton. En effet, dans la zone d'étude, la plupart des intrants organiques sont confectionnés par les producteurs qui sont accompagnés des structures en charge de la production biologique. Ainsi, les charges qui sont liées à la confection de ces intrants ne sont prises en compte dans l'évaluation économique faite par les producteurs. De plus, la production biologique du coton fait intervenir des prémiums qui sont payés aux producteurs lors de la commercialisation du coton. L'ajout de ce prémium au prix de vente du coton biologique rend sa culture plus rentable que celle du coton conventionnel aux yeux des producteurs.

Pour ce qui est du rendement, il ressort de l'analyse de la Figure 10, que les rendements sont plus élevés au niveau de la production conventionnelle. En effet, selon leur expérience, les producteurs conventionnels détiennent les rendements de production les plus élevés. Ceci pourrait s'expliquer par l'apport non négligeable des engrais chimiques.

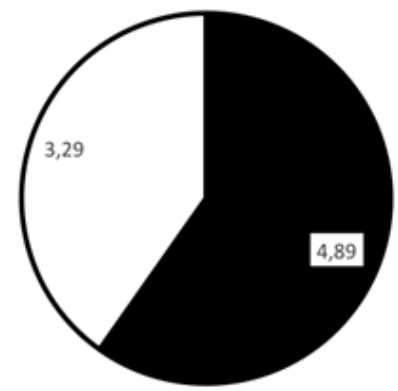

- Poids critère biol ogi que

DPoids critère conventionnei

Figure 7 : Analyse comparée des effets sur la création d'emplois des modes de production biologique et conventionnel du coton 


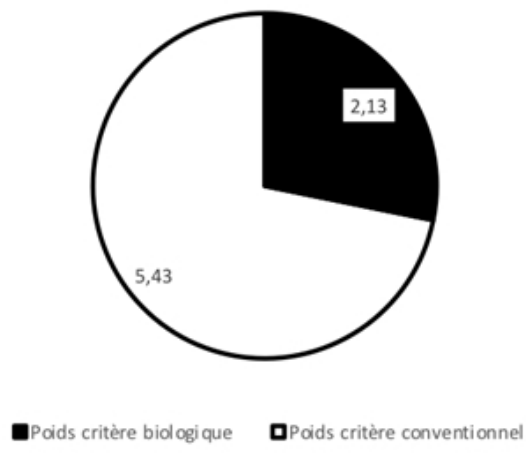

Figure 8 : Analyse comparée des effets sur le coût de production des modes de production biologique et conventionnel du coton

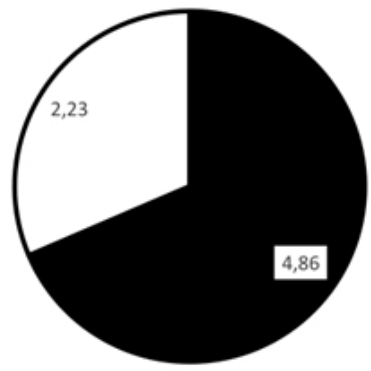

Poids critère biol ogi que

Doids critère conventionnel

Figure 9 : Analyse comparée des effets sur le coût de récolte des modes de production biologique et conventionnel du coton

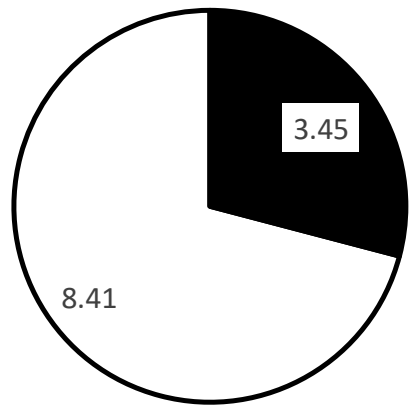

Poids critère biologique $\boldsymbol{\square}$ Poids critère conventionnel

Figure 10 : Analyse comparée des effets sur le rendement des modes de production biologique et conventionnel du coton 


\section{Conclusion}

Cette étude comparative des modes de production de coton conventionnel et biologique révèle sur le plan environnemental que le mode biologique de production de coton a obtenu la plus forte cotation. Ainsi, les producteurs reconnaissent que la production biologique assure mieux la protection et la conservation de l'environnement comparativement à la production conventionnelle. Au plan social, les résultats ont montré que le mode de production biologique présente moins d'effet négatif sur la santé des producteurs. Cependant, la production biologique nécessite beaucoup de temps de travail de la part des producteurs. Ceci constitue une limite à l'extension des superficies des cultures biologiques. Au plan économique, les producteurs estiment que le mode conventionnel de production de coton donne un rendement assez élevé et permet aux producteurs d'avoir aussi un revenu assez important comparativement à la production biologique. Cependant, ils reconnaissent également que le mode conventionnel de production de coton nécessite un coût de production assez élevé à comparer à celui du mode biologique.

De l'analyse des deux modes de production sur les trois précédents plans, on observe que le mode de production de coton conventionnel a obtenu le plus faible résultat (-59) tandis que le mode biologique détient le plus fort résultat $(+37)$. Par conséquent, la production biologique de coton reste le meilleur mode au vu des paramètres considérés dans l'étude. Les résultats ainsi obtenus avec l'analyse multicritère peuvent être discutables et peuvent aussi varier en fonction des critères sélectionnés, de la perception des enquêtés et de la connaissance des chercheurs. Toutefois, ces résultats peuvent servir de boussole dans les politiques de promotion d'une agriculture durable, surtout dans le contexte particulier des changements climatiques.

\section{References:}

1. Assogba CG. (2014). Représentations de l'environnement et adoption des pratiques durables de production par la coton culture du Bénin. Thèse de doctorat, Université de Liège/Gembloux Agro-Bio Tech, Belgique, $204 \mathrm{p}$.

2. Berti F., Hof JL., Zagbaï HS., Lebailly P. (2006). Le coton dans le Monde, place du coton africain et principaux enjeux. In Biotechnol. Agron.Soc.Environ. 10(4), 271-280.

3. Crowder, D. W., Northfield, T.D., Gomulkiewicz, R., Snyder, W. E. (2012). Conserving and promoting evenness: organic farming and firebased wildland management as case studies. Ecology, 93(9) : 20012007.

4. Dagbenonbakin GD., Chougourou CD., Ahoyo Adjovi NR., Fayalo G., Djenontin JPA., Igue AM. (2012). Effets agronomiques du 
compost et du N14P23K14S5B1 sur la production et les caractéristiques du rendement de coton-graine au Nord Bénin. Bulletin de la Recherche Agronomique du Bénin (BRAB), Numéro spécial Coton, Septembre 2012.

5. De Schutter, O. (2011). How not to think of land-grabbing: three critiques of large-scale investments in farmland. Journal of Peasant Studies 38(2):249-279.

6. Dossa F., Miassi Y.(2018). Facteurs Socio-Economiques Influençant L'adoption de Coton Biologique au Nord-Est du Bénin: Cas de la Commune de Kandi. International Journal of Progressive Sciences and Technologies (IJPSAT), 2018, 6 (2), pp.577-584.

7. Faq-logistique. (2019). L'analyse multicritère. In Faq-logistique. Multicritères. https://www.faq-logistique.com/Multicriteres.htm (Consulté: 23/10/2019).

8. Glin LC., Mol APJ., Oosterveer P., Vodouhe DS. (2012). Governing the transnational organic cotton network from Benin. Global Network, 12(3), 333-354.

9. Glin LC., Kuiseau J., Thiam A., Vodouhe DS., Dinham B., Ferrigno S. (2006). Living with Poison: Problems of Endosulfan in West Africa Cotton Growing Systems. Pesticide Action Network UK, London.

10. Jouffret, P. Labalette, F., Parachini, E. (2015). Analyse multicritère de la production de soja dans des exploitations agricoles contrastées du Sud-Ouest de la France. OCL, 22 (5): 1-12.

11. Lacasse C. (2016). Analyse comparative de quatre modes d'agriculture du blé au Québec : intensive, conventionnelle, raisonnée et biologique. Essai présenté au Centre universitaire de formation en environnement et développement durable (CUFE) en vue de l'obtention du grade de maître en environnement (M. Env.).

12. Lampkin N., Measures M. (2001). Organic Farm Management Handbook: Organic Farming Research Unit. Institute of Rural Studies, University of Wales, Aberystwyth.

13. Mader P., Fließbach A., Dubois D., Gunst L., Fried P., Niggli U. (2002). Soil fertility and biodiversity in organic farming. Science 296, 1694-1697.

14. MAEP (2017). Plan Stratégique du Développement du Secteur Agricole (PSDSA), Ministère de l'Agriculture, de l'Elevage et de la Pêche (MAEP).

15. Matthess A., van den Akker E., Chougourou D., Midingoyijun S. (2005). Le coton au Bénin : Compétitivité et durabilité de cinq systèmes culturaux cotonniers dans le cadre de la filière. Deutsche Gesellschaft für Technische Zusammenarbeit $(\mathrm{GmbH})$ Coopération 
Technique Al lemande. Imprimerie Minute, 03 B. p. 1354, Cotonou, Bénin.

16. Mena SB. (2000). Introduction aux méthodes multicritères d'aide à la Décision. Biotechnol. Agron. Soc. Environ. 20004 (2), 83-93.

17. New Agriculturalist (2008). Country Profile - Benin. Disponible sur http://www.new-ag.info/en/country/profile.php?a=389 (dernière consultation: 23/04/2019).

18. OBEPAB (2002). Le Coton au Benin: rapport de consultation sur le coton conventionel et le coton biologique au Benin. PAN UK's Pesticides, Poverty and Livelihoods project.

19. OBEPAB (2013). Rapport de mission de formation en Ethiopie sur : Utilisation de l'aliment complémentaire (Bénin Food) pour lutter contre les ravageurs du cotonnier.

20. OCEE (2008). La boussole bernoise du développement durable. In Office de la coordination environnementale et de l'énergie (OCEE). La direction. Portrait de la direction. Dossiers. Développement durable. Évaluation de la durabilité.

21. Oehl, F., Sieverding, E., Mäder, P., Dubois, D., Ineichen, K., Boller, T., Wiemken, A. (2004). Impact of long-term conventional and organic farming on the diversity of arbuscular mycorrhizal fungi. Oecologia, 138 (4): $574-583$.

22. Pimentel, D., Hepperly, P., Hanson, J., Douds, D., Seidel, R. (2005). Environmental, Energetic, and Economic Comparisons of Organic and Conventional Farming Systems. BioScience, 55 (7): 573 - 582.

23. Pretty J., Toulmin C., Williams S. (2011). Sustainable intensification in African agriculture. International Journal of Agricultural Sustainability 9: 5-24.

24. Reganold JP., Papendick RI., Parr JF. (1990). Sustainable agriculture. Scientific American 262, 112-120.

25. Reganold P., Jackson-Smith D., Batie SS., Harwood RR., Kornegay JL., Bucks D., Willis P. (2011). Transforming US agriculture, Science, 332 (6030) (2011), pp. 670-671

26. Savadogo PW., Traoré O., Topan M., Tapsoba KH., Sedogo PM, Bonzi-Coulibaly LY. (2006). Variation de la teneur en résidus de pesticides dans les sols de la zone cotonnière du Burkina- Faso. Journal Africain des Sciences de l'Environnement, Numéro 1, 29-39p.

27. Sodjinou E., Glin LC., Nicolay G.,Tovignan S., Hinvi J. (2015). Socioeconomic determinants of organic cotton adoption in Benin, West Africa. Agric. Food Econ., vol. 3, no 12, p. 22, 2015.

28. Traoré O. (2008). Les succès de la lutte intégrée contre les ravageurs du cotonnier en Afrique de l'Ouest. 67ème réunion plénière de l'ICAC. Ouagadougou (Burkina Faso), 16-21 novembre 2008, 11 p. 
29. Trepanier K. (2015). Analyse environnementale et socio-économique de la production de canneberges au Québec en fonction des principes de développe. Essai présenté au Centre universitaire de formation en environnement et développement durable. Université de Sherbrooke.

30. Vognan G., Glin L., Bamba I., Ouattara BM., Nicolay G. (2017). Analyse comparative de la rentabilité des systèmes de production de coton biologique, conventionnel et transgénique au Burkina Faso, p. 12-24. Tropicultura, 2017 vol. $35 \mathrm{~N}^{\circ} 1$.

31. Westerberg V. (2017). L'économie de la production de coton conventionnel et biologique. Une étude de cas de la commune de Banikoara au Bénin. Rapport soumis à l'Initiative de l'économie de la dégradation des terres.

32. Zoundji C G. (2010). Are women behind the shift to organic agriculture? In Puzzles for innovation: Case studies from Asia and Africa. Book edited by ILEIA, Centre for Learning on Sustainable Agriculture Wageningen, the Netherlands. 